\title{
Bioinformatic analysis of changes in RNA polymerase II transcription stimu- lated by estradiol in MCF7 cells
}

\author{
J. WU', ${ }^{1,}, \mathrm{CAI}^{2}$, G. ZHAO \\ ${ }^{1}$ MOE Key Laboratory of Hydrodynamics and School of Naval Architecture, Ocean and Civil Engineering, Shanghai Jiao Tong University, Shang- \\ hai, 200240, China; ${ }^{2}$ School of Biological Science and Medical Engineering, Southeast University, Nanjing, Jiangsu, 210096, China; ${ }^{3}$ School of \\ Medical Instrument and Food Engineering, University of Shanghai for Science and Technology, Shanghai, 200093, China
}

${ }^{*}$ Correspondence: wujie2016wj@hotmail.com

Received December 14, 2016 / Accepted April 10, 2017

\begin{abstract}
Estradiol (E2) is the most potent estrogen and RNA polymerase II (Pol II) regulates a great mass of gene expression. This study was designed to illustrate the mechanisms of estrogen-dependent human breast cancer (BC) through Pol II. ChIP-seq data, DNase-seq data and other sequencing data of human BC MCF-7 cells were downloaded from Gene Expression Omnibus database. Each of these datasets included one control and one E2 treated sample. Sequence alignment was performed and Pol II factor binding signal was determined. Functional enrichment analysis of particular genes was performed, along with transcription factor (TF) motif enrichment analysis. Sites with enhanced Pol II binding were identified in intergenic regions. Pol II binding sites with increased binding signal facilitated chromosome switching from a closed to an open state. A total of 59 TFs, including KLF4 (Kruppel-like factor 4), were identified. Besides, enrichment analysis revealed that protein synthesis and metabolic processes as well as cell cycle processes were highlighted. The KLF4 motif was found to be enriched and was significantly enhanced in the promoter region for Pol II binding. Cell cycle processes, protein synthesis and metabolism play critical roles in the progression of E2-stimulated BC. In addition, KLF4 may be important for the progression.
\end{abstract}

Key words: estradiol, breast cancer, RNA polymerase II, transcription factors

Breast cancer (BC) is characterized by lumps in the breast tissue, changes in breast shape, skin dimpling, fluid secretion through the nipples, or swollen lymph nodes, bone pain, yellow skin, and shortness of breath [1]. Globally, BC affects approximately $12 \%$ of women and is the most frequent invasive cancer in women [2]. BC resulted in 458,503 deaths worldwide in 2008 [3], and it is alone expected to account for $29 \%$ of all new cancers in women in 2015[4]. Estradiol (E2), or more precisely, $17 \beta$-estradiol, is the predominant hormone secreted by the ovaries. It affects many tissues, including bone [5], and is essential for the development and maintenance of female reproductive tissues [6]. E2 is widely known to have proliferative effects on estrogen-dependent cancer cells, and antiestrogens are known to inhibit this effect.

Hormone-dependent BC, which is also known as estrogen-dependent BC, requires estrogen to sustain tumor growth. Key et al. found that [7] the risk of BC increases significantly with increasing concentrations of several hormones, including E2. Cauley et al. [8] reported that elevated serum E2 is a strong risk factor for BC. However, the precise mechanism by which $\mathrm{E} 2$ affects $\mathrm{BC}$ is complex and is controlled by multiple factors. For example, in BC cells, E2 can regulate the expression and secretion of tumor necrosis factor- $\alpha$ [9], which is closely linked to BC growth [10]. All cancers, which show dysregulated DNA transcription and cellular growth, are regulated by various transcription-related factors. RNA polymerase II (Pol II) is an elongation factor that synthesizes precursors of mRNAs and most small nuclear RNAs and microRNAs. It requires a range of transcription factors (TFs) for binding to promoters [11]. In addition, the Pol II core promoter and TFs are key components in the regulation of gene expression. Therefore, the process of tumor progression in E2-dependent BC is likely to be also influenced by Pol II and associated TFs.

In the current study, we measured and compared dynamic changes in the intensity of Pol II binding in human BC MCF7 cells. Based on these results, we identified candidate genes involved in the degradation process of the E2 stimulus 
in MCF7 cells. Finally, using the promoter regions of these genes, transcriptional regulation elements associated with E2 stimulus were screened using TF motif enrichment analysis. This study identified key genes and biological processes involved in the proliferation of cancerous MCF7 cells stimulated by E2.

\section{Materials and methods}

Microarray datasets. ChIP-seq data (accession number: GSE23701) [12] generated using a GPL9115 Illumina Genome Analyzer II (Homo sapiens) platform were downloaded from the Gene Expression Omnibus (GEO) database. This dataset was collected by Joseph et al. [12], using samples with E2 stimulation or without stimulation, using the vehicle as a control. Fragments were isolated using formaldehydeassisted isolation of regulatory elements (FAIRE) and then deep sequenced. From this dataset, we used Pol II sequence data from E2-treated MCF-7 cells (GSM588576) and non-treated (DMSO as vehicle) MCF-7 cells (GSM588577).

DNase-seq data (accession number: GSE33216) collected by $\mathrm{He}$ et al. [13] were also included. The selected data were from samples of MCF-7 cells treated with E2 (GSM822390) or not (GSM822389), collected using an Illumina Genome Analyzer (Homo sapiens) GPL9052.

Sequencing data (accession number: GSE43070) deposited into the GEO database by Jin et al. [14] were also included. Data were collected using a GPL10999 (Illumina Genome Analyzer IIx (Homo sapiens) or a GPL11154 (Illumina HiSeq 2000 Homo sapiens) platform. MCF-7 cells were treated with E2 (GSM1154040) or without reagent (GSM1154039).

Sequence alignment. The reads for the Pol II ChIPseq and DNase-seq data were aligned against the human reference genome (UCSC hg19) using Bowtie software version 0.12 .9 [15]. Only unique matches with a mismatch number $\leq 2$ were included. Only reads that met these criteria were used for subsequent analysis.

TopHat [16], as an efficient read-mapping algorithm, was applied to align RNA-Seq reads. Default parameter settings were used, and only reads uniquely matched to the genome with $\leq 2$ mismatches were included. Combined with reference sequence (RefSeq) gene annotation, transcript assembly and expression estimation were performed with the Cuffdiff tool in the Cufflinks package [17], based on fragments per transcript kilobase per million fragments mapped (FPKM).

Determination of Pol II factor binding sites. The SelfAdministration of Medication tool (SAMtools) [18] was employed to remove potential PCR duplicates form the Pol II ChIP-seq data. When multiple reads mapped to the same locus, flattened operation was adopted to ensure that the number of reads was $\leq 1$. With a sliding 100 -bp window, modification levels were quantified by counting the number of reads (extended to $100 \mathrm{bp}$ both up- and down-stream) that overlapped with a target region. The Model-based Analysis of ChIP-seq (MACS) algorithm with the MACS software version 1.4.0 [19] was used to identify enriched regions (peak calling), with $\mathrm{p}<0.00001$.

Analysis of changes in Pol II binding signal. Normalized reads per million (RPM) analysis was performed to normalize read depth and number. Differential analysis of the degree of enrichment of each peak between samples was performed with Noise Sensitivity Questionnaire (NOISeq) software, with a significance threshold of $\mathrm{q} \geq 0.8$ [20].

Functional enrichment analysis. The Database for Annotation, Visualization, and Integrated Discovery (DAVID) [21] is commonly used for systematic and integrative analysis of large sets of genes and proteins. In the current study, Gene Ontology (GO) - biological process (BP) and Kyoto Encyclopedia of Genes and Genomes (KEGG) pathway analyses were conducted using DAVID. Default parameter settings were used.

Transcription factor (TF) motif enrichment analysis. For motif discovery, the region from $1000 \mathrm{bp}$ upstream to 500 bp downstream of the transcription start site (TSS) was scanned using the Seqpos [22] algorithm. TF-enriched motifs with $\mathrm{p}<0.001$ and $\mathrm{Z}$-score $<-10$ were selected.

\section{Results}

Pol II binding sites with differential signal intensity. Based on the ChIP-seq data, 37,844 Pol II binding sites with high signal intensity were identified. A total of 7812 (20.64\%) different binding sites were identified, including 5753 that showed a significant increase and 2059 that showed a significant decrease after E2 stimulus.

The distribution of E2 stimulus-related Pol II binding sites was analyzed. Binding site peaks were mainly located in the intergenic regions, for those with increased and reduced binding intensity, and those in promoter regions were the next most common.

Effect of changes in chromosome structure on Pol II binding. Figure 1 shows that no significant changes in DNase I hypersensitive site (DHS) signal were observed around the sites that showed decreased Pol II binding following E2 stimulation. Moreover, regardless of the E2 stimulus, regions around Pol II binding sites were more open than the flanking regions. However, the reduced binding ability of Pol II did not result in switching of the regional chromosome structure from an open to a closed state. In contrast, DHS signal was enhanced in the sites that showed increased Pol II binding following E2 stimulation, and the chromosome structure showed a more open state than prior to E2 stimulus. Further analysis of binding sites in promoters around the sites that showed increased Pol II binding showed significant transition to the open state.

Annotation and functional analysis of differentially binding regions. Adjacent genes were identified by annotating promoter regions that contained different Pol II binding signals. These 1727 sites with increased signal corresponded to 1696 genes including 1520 encoding genes and 

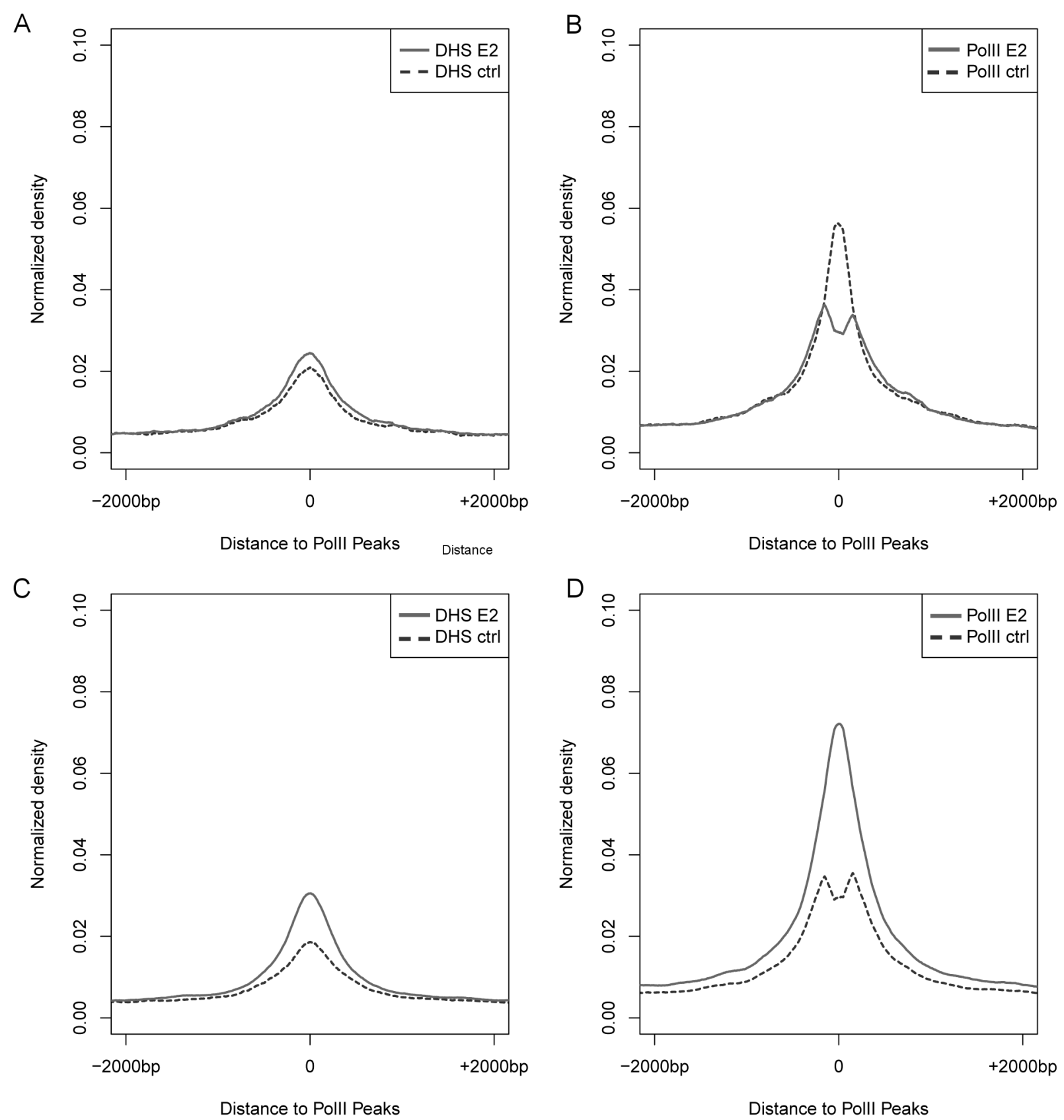

Figure 1. RNA polymerase II (Pol II) and DNase I hypersensitive site (DHS) signal near Pol II binding sites that showed differential binding affinity before and after estradiol (E2) stimulus. The upper and lower figures show the distribution of sites with reduced and increased Pol II binding after E2 stimulus, respectively. The left and right figures show the distribution of DHS and Pol II signal before and after E2 stimulus, respectively. The solid and dashed curves indicate signal before and after stimulus, respectively.

176 that encoded non-coding RNAs. In the 1520 sites that encoded genes, 59 were identified TFs. Further analyses revealed that some of these genes were tumor-related, such as KLF4 (Kruppel-like factor 4), MAFB (v-maf avian musculoaponeurotic fibrosarcoma oncogene homolog B), and MUM1 (melanoma associated antigen 1). The 535 sites with reduced Pol II binding corresponded to 526 genes. Genes including
BC suppressor genes such as BRCA2 (breast cancer 2, early onset) and tumor-suppressor transcription factors, $C T B P 1$ (C-terminal binding protein 1), NR4A1 (nuclear receptor subfamily 4, group A, member 1), SMARCB1 (SWI/SNF related, matrix associated, actin dependent regulator of chromatin, subfamily b, member 1), and SOX11 (SRY, sex determining region $\mathrm{Y}$ - box 11) were identified. In addition, 
526 genes including 470 encoding genes (17 TFs) and 56 encoding non-coding RNAs, were identified among the 535 Pol II binding sites with decreased signal.

GO enrichment analysis (Table 1) revealed that genes in the region with increased binding signal were mainly protein trafficking-related, such as protein targeting and protein transport genes. Genes in the region with decreased binding signal mainly showed cell cycle enrichment. However, this result was not significant $(\mathrm{p}<0.01, \mathrm{FDR}>0.05)$.

KEGG pathway enrichment analysis of genes in the region with increased binding signal showed that these genes were mainly involved in ubiquitin mediated proteolysis, Fc gamma R-mediated phagocytosis, and MAPK (mitogenactivated protein kinase) signaling pathway etc. (Table 2). In particular, 11 genes were related to cell cycle pathways. No pathways were found to be associated with genes in the region with decreased binding signal.

TF motif enrichment analysis. A total of 10 significantly enriched TFs were identified in the promoter region

Table 1. Functional enrichment analysis of RNA polymerase II binding regions responded genes.

\begin{tabular}{|c|c|c|c|c|}
\hline & Term & Count & p-value & FDR \\
\hline \multirow{8}{*}{ 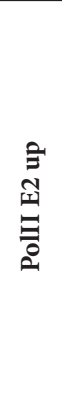 } & GO:0006605 protein targeting & 38 & $1.25 \mathrm{E}-06$ & 0.002278595 \\
\hline & GO:0015031 protein transport & 93 & $1.98 \mathrm{E}-06$ & 0.003609962 \\
\hline & GO:0017038 protein import & 27 & $3.30 \mathrm{E}-06$ & 0.006015974 \\
\hline & $\begin{array}{l}\text { GO:0046907 intracellular } \\
\text { transport }\end{array}$ & 82 & $3.76 \mathrm{E}-06$ & 0.006861461 \\
\hline & GO:0008104 protein localization & 103 & $3.81 \mathrm{E}-06$ & 0.006937147 \\
\hline & $\begin{array}{l}\text { GO:0006913 nucleocytoplasmic } \\
\text { transport }\end{array}$ & 29 & 1. & 948 \\
\hline & GO:0051169 nuclear transport & 29 & $1.37 \mathrm{E}-05$ & 0.024956618 \\
\hline & $\begin{array}{l}\text { GO:0033365 protein localization } \\
\text { in organelle }\end{array}$ & 27 & $2.52 \mathrm{E}-05$ & 0.0459067 \\
\hline \multirow{3}{*}{ 坴 } & GO:00 & 35 & $1.98 \mathrm{E}-04$ & 0.334412741 \\
\hline & GO:0022402 cell cycle pro & 28 & $2.41 \mathrm{E}-04$ & 0.407175908 \\
\hline & GO:0000279 M phase & 19 & $5.71 \mathrm{E}-04$ & 0.961234423 \\
\hline
\end{tabular}

Count: the enriched gene number. GO: gene ontology. FDR: False Discovery Rate.

Table 2. Pathway enrichment analysis of increased RNA polymerase II signal sites corresponding genes.

\begin{tabular}{lcll}
\hline Term & Count & p-value & FDR \\
\hline $\begin{array}{l}\text { hsa04120:Ubiquitin mediated } \\
\text { proteolysis }\end{array}$ & 24 & $8.81 \mathrm{E}-05$ & 0.107648451 \\
$\begin{array}{l}\text { hsa04110:Cell cycle } \\
\text { hsa04666:Fc gamma R-mediated } \\
\text { phagocytosis }\end{array}$ & 20 & 0.001276895 & 1.549729414 \\
$\begin{array}{l}\text { hsa04010:MAPK signaling } \\
\text { pathway }\end{array}$ & 28 & 0.037255899 & 37.1307681 \\
$\begin{array}{l}\text { hsa00510:N-Glycan biosynthesis } \\
\text { hsa04660:T cell receptor signaling } \\
\text { pathway }\end{array}$ & 8 & 0.04051981 & 39.68717661 \\
\hline
\end{tabular}

Count: the enriched gene number. FDR: False Discovery Rate. with increased binding signal (Table 3). The identified genes included E2F1 (E2F transcription factor 1), EGR1 (early growth response 1), and KLF4, which were all cancer-related. However, KLF4 was not only enriched in the region with increased binding signal, but was also significantly enhanced in the promoter region.

The combined expression profile data showed that only KLF4 was up-regulated, and the other 9 TFs in the regions

Table 3. Motif enrichment analysis of transcription factor of different RNA polymerase II signal region responding genes.

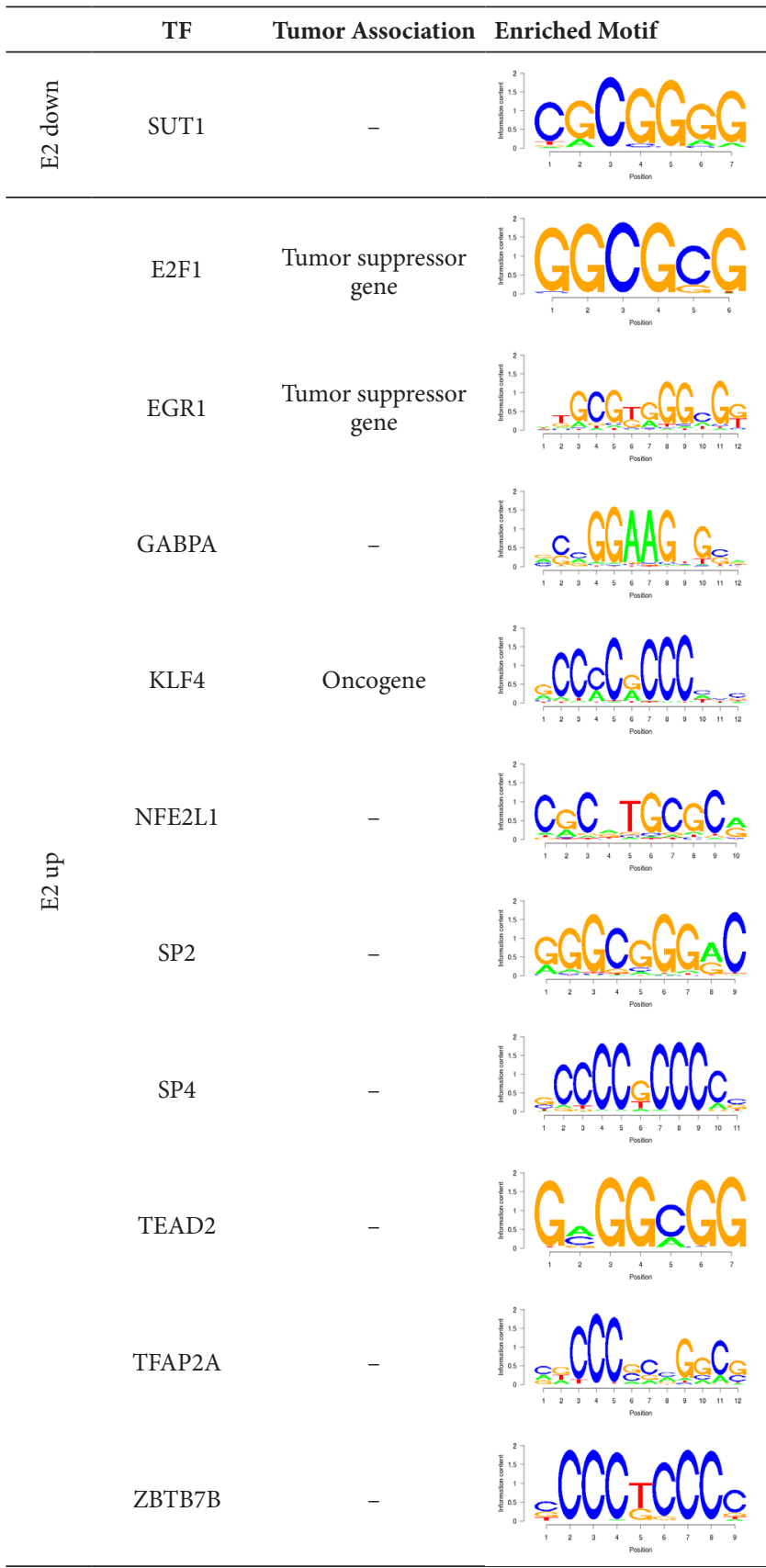

TF: transcription factors. E2: estradiol stimulated 


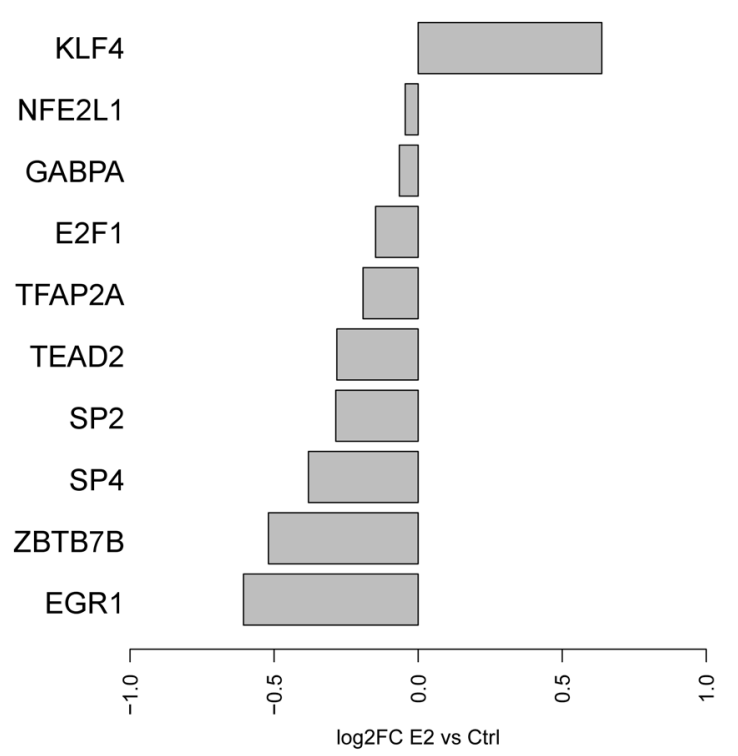

Figure 2. Changes in expression of 10 sites significantly enriched for transcription factor motifs at sites with increased RNA polymerase II signal. FC: fold change. E2: estradiol stimulated. Ctrl: control.

with increased Pol II signal were down-regulated to varying degrees (Figure 2). Out of the genes at sites with decreased Pol II signal, only SUT1 (solute carrier family 13, sodium/ sulfate symporter, member 4) showed significant TF motif enrichment.

\section{Discussion}

The mechanism underlying the development of estrogendependent BC is complex and remains unclear, and many further studies are needed. In the current study, three different microarray datasets (ChIP-seq, DNase-seq, and other sequencing data) collected for the human BC cell line MCF7 were analyzed. Sites with peaks of Pol II binding were mainly located in intergenic regions. A total of $59 \mathrm{TFs}$, including KLF4 and MAFB, were identified. KLF4 was TF motif-enriched and showed significantly enhanced binding signal in the promoter region of Pol II. In addition, GO and pathway enrichment analyses revealed that protein synthesis and metabolic processes, as well as cell cycle processes, may be important for the progression of E2-stimulated BC.

E2 affects gene expression by binding to E2 receptors in the cytosol, followed by translocation to the nucleus. Since it alters the Pol II binding signal, E2 affects Pol II binding efficiency and regulates expression of genes [23]. Because Pol II mainly acts on exon regions for transcription and the RNA Pol II core promoter is a key component in the regulation of gene expression [24], we chose to focus on genes transcribed by Pol II. In addition, changes in DHS signal revealed activation of gene expression, which helped to identify E2 stimulusrelated transcriptional activation of genes, combined with
Pol II binding signal. The subsequent functional enrichment results suggested that functions related to protein synthesis and metabolism, including protein targeting, protein transport, and ubiquitin mediated proteolysis, were significantly enriched. Zhang et al. suggested that proteins involved in metabolic pathways are highly expressed in HER-2/ neu-positive BC [25]. The role of the ubiquitin-proteasome system in neoplastic disease has been reported [26]. Therefore, E2, as the most potent form of estrogen, could contribute to the development of $\mathrm{BC}$ through protein transport, location, and metabolism. In addition, genes transcribed by enhanced Pol II binding were also enriched in the cell cycle pathway. These results are consistent with those reported in previous studies, which illustrated that cell proliferation is accelerated in estrogen-dependent BC $[27,28]$. Thus, E2-stimulated BC is initiated by mechanisms including protein transport and metabolism and the cell cycle.

Several genes were highlighted in this study. KLF4, which encodes Kruppel-like factor 4, regulates the expression of key TFs during embryonic development [29]. KLF4 expression was found to be up-regulated and corresponded to the sites with increased Pol II binding signal. In addition, KLF4 was TF motif-enriched in sites with increased Pol II binding signal. A previous study found that KLF4 is both an oncogene and a tumor suppressor gene [30]. In estrogen-dependent BC, KLF4 may inhibit the transcriptional activity of estrogen receptor-alpha $(\mathrm{ER} \alpha)$ and is transcriptionally activated by p53 [31]. Through regulation of the expression of manganese superoxide dismutase (MnSOD) and KLF4, estrogen plays an antiproliferative role in vascular smooth muscle cells [32]. A previous study demonstrated that KLF4 inhibits the growth of estrogen-dependent BC by suppressing ERa activity [31]. GO-BP enrichment analysis showed that KLF4 had dual regulatory functions such as negative and positive regulation of cell proliferation. These results indicate the complicated regulatory mechanism of cell proliferation. Based on the results described above, KLF4 may play important roles in the progress of $\mathrm{BC}$ development following E2 stimulus.

\section{Conclusion}

Analysis of Pol II binding signal revealed changes in the expression of genes, including those related to the cell cycle as well as protein transport, location, and metabolism, which play critical roles in the progression of E2-related BC. In addition, genes such as KLF4 that perform dual regulatory functions in processes such as cell proliferation were found to be up-regulated in E2-stimulated BC. These results may help to elucidate the complex regulatory mechanism of cell proliferation.

Acknowledgements: This work was supported by the National Natural Science Foundation of China (No.11572200, No. 11102113, No.11502146, No.11302050) and the National Basic Research Program of China (973 Program)(No.2013CB733800). 


\section{References}

[1] KATAJA V,CASTIGLIONEM,ESMOGUIDELINESWORKING GROUP. Primary breast cancer: ESMO clinical recommendations for diagnosis, treatment and follow-up. Ann Oncol. 2009; 20 Suppl 4:10-14. doi: 10.1093/annonc/mdp114

[2] MCGUIRE A, BROWN JAL, MALONE C, MCLAUGHLIN R, KERIN MJ. Effects of age on the detection and management of breast cancer. Cancers (Basel) 2015; 7: 908-929. doi: $10.3390 /$ cancers7020815

[3] DESANTIS CE, FEDEWA SA, GODING SAUER A, KRAMER JL, SMITH RA et al. Breast cancer statistics, 2015: Convergence of incidence rates between black and white women. CA Cancer J Clin 2016; 66: 31-42. doi: 10.3322/caac. 21320

[4] MOHAMMADBEIGI A, MOHAMMADSALEHI N, VALIZADEH R, MOMTAHENI Z, MOKHTARI M et al. Lifetime and 5 years risk of breast cancer and attributable risk factor according to Gail model in Iranian women. J Pharm Bioallied Sci 2015; 7: 207-211. doi: 10.4103/0975-7406.160020

[5] LAI S, COLLINS BC, COLSON BA, KARARIGAS G, LOWE DA. Estradiol modulates myosin regulatory light chain phosphorylation and contractility in skeletal muscle of female mice. Am J Physiol Endocrinol Metab 2016; 310: E724-733. doi: 10.1152/ajpendo.00439.2015

[6] RYAN KJ. Biochemistry of aromatase: significance to female reproductive physiology. Cancer Res 1982; 42: 3342s-3344s.

[7] KEY T, APPLEBY P, BARNES I, REEVES G, ENDOGENOUS HORMONES AND BREAST CANCER COLLABORATIVE GROUP. Endogenous sex hormones and breast cancer in postmenopausal women: reanalysis of nine prospective studies. J Natl Cancer Inst 2002; 94: 606-616.

[8] CAULEY JA, LUCAS FL, KULLER LH, STONE K, BROWNER W et al. Elevated serum estradiol and testosterone concentrations are associated with a high risk for breast cancer. Ann Intern Med 1999; 130: 270-277.

[9] TO SQ, CHEUNG V, LAZARUS KA, KNOWER KC, CLYNE CD. Estradiol regulates Tumor Necrosis Factor- $\alpha$ expression and secretion in Estrogen Receptor positive breast cancer cells. Mol Cell Endocrinol 2014; 394: 21-28. doi: 10.1016/j. mce.2014.06.020

[10] YU M, ZHOU X, NIU L, LIN G, HUANG J et al. Targeting transmembrane TNF- $\alpha$ suppresses breast cancer growth. Cancer Res 2013; 73: 4061-4074. doi: 10.1158/0008-5472. CAN-12-3946

[11] CARTHEW RW, CHODOSH LA, SHARP PA. An RNA polymerase II transcription factor binds to an upstream element in the adenovirus major late promoter. Cell 1985; 43: 439-448.

[12] JOSEPH R, ORLOV YL, HUSS M, SUN W, LI KONG S et al. Integrative model of genomic factors for determining binding site selection by estrogen receptor- $\alpha$. Mol Syst Biol 2010; 6: 456. doi: 10.1038/msb.2010.109

[13] HE HH, MEYER CA, CHEN MW, JORDAN VC, BROWN $\mathrm{M}$ et al. Differential DNase I hypersensitivity reveals factordependent chromatin dynamics. Genome Res 2012; 22: 1015-1025. doi: 10.1101/gr.133280.111

[14] JIN F, LI Y, DIXON JR, SELVARAJ S, YE Z et al. A high-resolution map of the three-dimensional chromatin interactome in human cells. Nature 2013; 503: 290-294. doi: 10.1038/nature12644
[15] LANGMEAD B, TRAPNELL C, POP M, SALZBERG SL. Ultrafast and memory-efficient alignment of short DNA sequences to the human genome. Genome Biol 2009; 10: R25. doi: 10.1186/gb-2009-10-3-r25

[16] TRAPNELL C, PACHTER L, SALZBERG SL. TopHat: discovering splice junctions with RNA-Seq. Bioinformatics 2009; 25: 1105-1111. doi: 10.1093/bioinformatics/btp120

[17] TRAPNELL C, WILLIAMS BA, PERTEA G, MORTAZAVI A, KWAN G et al. Transcript assembly and quantification by RNA-Seq reveals unannotated transcripts and isoform switching during cell differentiation. Nat Biotechnol 2010; 28: 511-515. doi: 10.1038/nbt.1621

[18] LI H, HANDSAKER B, WYSOKER A, FENNELL T, RUAN $\mathrm{J}$ et al. The sequence alignment/map format and SAMtools. Bioinformatics 2009; 25: 2078-2079. doi: 10.1093/bioinformatics/btp352

[19] ZHANG Y, LIU T, MEYER CA, EECKHOUTE J, JOHNSON DS et al. Model-based analysis of ChIP-Seq (MACS). Genome Biol 2008; 9: R137. doi: 10.1186/gb-2008-9-9-r137

[20] TARAZONA S, GARC A-ALCALDE F, DOPAZO J, FERRER A, CONESA A. Differential expression in RNA-seq: a matter of depth. Genome Res 2011; 21: 2213-2223. doi: 10.1101/gr.124321.111

[21] HUANG DA W, SHERMAN BT, LEMPICKI RA. Systematic and integrative analysis of large gene lists using DAVID bioinformatics resources. Nat Protoc 2008; 4: 44-57. doi: 10.1038/nprot.2008.211

[22] HE HH, MEYER CA, SHIN H, BAILEY ST, WEI G et al. Nucleosome dynamics define transcriptional enhancers. Nat Genet 2010; 42: 343-347. doi: 10.1038/ng.545

[23] MUSE GW, GILCHRIST DA, NECHAEV S, SHAH R, PARKER JS et al. RNA polymerase is poised for activation across the genome. Nat Genet 2007; 39: 1507-1511.

[24] JUVEN-GERSHON T, HSU J-Y, THEISEN JW, KADONAGA JT. The RNA polymerase II core promoter-the gateway to transcription. Curr Opin Cell Biol 2008; 20: 253-259. doi: 10.1016/j.ceb.2008.03.003

[25] ZHANG D, TAI LK, WONG LL, CHIU LL, SETHI SK et al. Proteomic Study Reveals That Proteins Involved in Metabolic and Detoxification Pathways Are Highly Expressed in HER-2/neu-positive Breast Cancer. Mol Cell Proteomics 2005; 4: 1686-1696.

[26] BHATTACHARJEE P, MAZUMDAR M, GUHA D, SA G. In Ubiquitin-Proteasome System in the Hallmark of the cancer, p 159-186. In: NJ. Dhalla, S. Chakraborti (Eds.). Role of Proteases in Cellular Dysfunction. Springer, New York, 2014, pp 462. ISBN 978-1-4614-9098-9.

[27] PRALL OW, ROGAN EM, SUTHERLAND RL. Estrogen regulation of cell cycle progression in breast cancer cells. J Steroid Biochem Mol Biol 1998; 65: 169-174.

[28] FALANY JL, MACRINA N, FALANY CN. Regulation of MCF-7 breast cancer cell growth by $\beta$-estradiol sulfation. Breast Cancer Res Treat 2002; 74: 167-176.

[29] KUO CT, LEIDEN JM. Transcriptional regulation of T lymphocyte development and function. Annu Rev Immunol 1999; 17: 149-187. 
[30] ROWLAND BD, PEEPER DS. KLF4, p21 and context-dependent opposing forces in cancer. Nat Rev Cancer 2006; 6: 11-23.

[31] AKAOGI K, NAKAJIMA Y, ITO I, KAWASAKI S, OIE S et al. KLF4 suppresses estrogen-dependent breast cancer growth by inhibiting the transcriptional activity of ERa. Oncogene 2009; 28: 2894-2902. doi: 10.1038/onc.2009
[32] SIVRITAS D, BECHER MU, EBRAHIMIAN T, ARFA O, RAPP $S$ et al. Antiproliferative effect of estrogen in vascular smooth muscle cells is mediated by Kruppel-like factor- 4 and manganese superoxide dismutase. Basic Research in Cardiology 2011; 106: 563-575. doi: 10.1007/s00395-0110174-Z. 